\title{
Tumor Mass Fragmentation
}

National Cancer Institute

\section{Source}

National Cancer Institute. Tumor Mass Fragmentation. NCI Thesaurus. Code C94526.

A finding indicating that a tumor mass has been divided into two or more tumors. 